\title{
Green Functions for Topology Change
}

\author{
Jérôme Martir* \\ Institut d'Astrophysique de Paris-GReCO, 98 bis Boulevard Arago, 75014 Paris, France \\ Nelson Pinto-Netd and Ivano Damião Soared \\ Laboratorio de Cosmologia e Fisica Experimental de Altas Energias, \\ Centro Brasileiro de Pesquisas Fisicas, Rua Dr. Xavier Sigaud 150, \\ Urca, Rio de Janeiro CEP 22290-180-RJ, Brazil
}

(Dated: October 25, 2018)

\begin{abstract}
We explicitly calculate the Green functions describing quantum changes of topology in FriedmanLemaitre-Robertson-Walker Universes whose spacelike sections are compact but endowed with distinct topologies. The calculations are performed using the long wavelength approximation at second order in the gradient expansion. We argue that complex metrics are necessary in order to obtain a non-vanishing Green functions and interpret this fact as demonstrating that a quantum topology change can be viewed as a quantum tunneling effect. We demonstrate that quantum topological transitions between curved hypersurfaces are allowed whereas no transition to or from a flat section is possible, establishing thus a selection rule. We also show that the quantum topology changes in the direction of negatively curved hypersurfaces are strongly enhanced as time goes on, while transitions in the opposite direction are suppressed

PACS numbers: $98.80 . \mathrm{Cq}, 98.70 . \mathrm{Vc}$
\end{abstract}

\section{INTRODUCTION}

Combining the principles of General Relativity with those of Quantum Mechanics seems necessary in order to understand more completely the physical nature of the gravitational field. As a result, new properties are expected to emerge. For instance, in classical General Relativity, topology changes are forbidden in the sense that their presence would necessarily imply the appearance of either singularities or closed timelike curves, a result known as the Geroch theorem, see Ref. 1]. On the other hand, topology changes are widely believed to become allowed if the quantum-mechanical nature of the gravitational field is taken into account. Studying this problem is notoriously known as a difficult technical task and in the literature only very general aspects of the issue have been discussed so far, see e.g. Refs. 2, 3]. Recently, a model where the theoretical ideas about topology change can be implemented concretely, at the level of equations, has been proposed in Ref. [4]. The metric of this model is given by a Friedmann-Lemaître-Robertson-Walker-like metric where the curvature of the spacelike sections, usually denoted as $\mathcal{K}$, is now a time dependent function, $\mathcal{K}=\mathcal{K}(t)$

$\mathrm{d} s^{2}=-N^{2}(t) \mathrm{d} t^{2}+a^{2}(t)\left\{\mathrm{d} \xi^{2}+\left[\frac{\sin (\sqrt{\mathcal{K}} \xi)}{\sqrt{\mathcal{K}}}\right]^{2} \mathrm{~d} \Omega^{2}(\theta, \varphi)\right\}$.

The three-dimensional spacelike hypersurfaces $\mathbb{V}^{3}$ of the model may be endowed with a large class of topologies

\footnotetext{
*Electronic address: jmartin@iap.fr

${ }^{\dagger}$ Electronic address: nelson@cbpf.br

${ }^{\ddagger}$ Electronic address: ivano@cbpf.br
}

compatible with such geometry. Technically, the metric has always the form displayed above (since it is locally defined) but the ranges of variations of the coordinates are modified. The scalar curvature of the metric is always constant (for fixed $t$ ) and equal to $6 \mathcal{K} / a^{2}$. We will restrict our considerations to compact (and orientable) spaces in order to avoid possible surface terms in the Hamiltonian formalism. We can show that any compact three manifold with constant curvature is homeomorphic to $\mathbb{V}^{3} / \Gamma$, where the universal covering $\mathbb{V}^{3}$ is either $\mathbb{R}^{3}$, $\mathbb{S}^{3}$ or $\mathbb{H}^{3}$, according to the sign of $\mathcal{K}(\mathcal{K}=0, \mathcal{K}<0$, $\mathcal{K}>0$, respectively). The group $\Gamma$ is the group of covering transformations. In three dimensions the closed (i.e. compact without boundary) space is now homeomorphic to a polyhedron the faces of which are identified by pairs, for a general review see Ref. [5].

Let us first consider the case $\mathcal{K}>0$. The universal covering is $\mathbb{S}^{3}$. Since it is compact, all the three-dimensional spaces admitting this universal covering are also compact. There is an infinite number of such spaces. We will only consider two cases: $\mathbb{S}^{3}$ itself and the Poincaré dodecahedral space, $\mathbb{D}^{3} \equiv \mathbb{S}^{3} / I^{*}$, where $I^{*}$ is the binary symmetry group of the icosahedron. The ranges of variation of the coordinates $(\xi, \theta, \varphi)$ can always be written as

$$
0 \leq \xi \leq \frac{\Xi\left(\theta, \varphi ; \mathbb{V}^{3}\right)}{\sqrt{\mathcal{K}}}, \quad 0 \leq \theta \leq \pi \quad 0 \leq \varphi \leq \pi
$$

The symbol $\mathbb{V}^{3}$ in the argument of the function $\Xi$ indicates that $\Xi\left(\theta, \varphi ; \mathbb{V}^{3}\right)$ is not the same function for different $\mathbb{V}^{3}\left(\mathbb{S}^{3}\right.$ or $\left.\mathbb{D}^{3}\right)$. Of course, the case $\mathbb{S}^{3}$ is very simple and we just have $\Xi\left(\theta, \varphi ; \mathbb{S}^{3}\right)=\pi$. This guarantees that no conical singularities will appear in this case. If $\mathbb{V}^{3}=\mathbb{D}^{3}$ the function $\Xi$ is also not arbitrary but its explicit expression is much more complicated. For the case $\mathcal{K}<0$, 
where the universal covering is $\mathbb{H}^{3}$, the classification of closed three-dimensional spaces is still an open question, but it is known that the volumes of the polyhedron are fixed as in the $\mathcal{K}>0$ case. Finally, the case $\mathcal{K}=0$ have six different tessellations by polyhedrons, some of them with arbitrary volume for their polyhedrons (for more details see again Ref. [5]). What is important for us here is that the topologies compatible with one $\mathcal{K}$ are not compatible with other $\mathcal{K}$ of distinct sign. Hence, a change in the sign of $\mathcal{K}$, or in the sign of the curvature scalar $6 \mathcal{K} / a^{2}$, necessarily indicates a change of topology. Our strategy here is to investigate whether this change is possible quantum mechanically.

The quantization of this model was carried out in Ref. [4]. In that article, it was shown that a Hamiltonian formulation necessarily requires a passage to a midisuperspace description. It is not possible to construct a minisuperspace Hamiltonian from the metric Eq. (11) because, as far as $\mathcal{K}$ depends on time, this metric does not represent a spatially homogeneous space-time, in the sense that the components of the four dimensional curvature tensor in a local frame are functions of $t$ and $\xi$. The existence of the non-vanishing component of the Ricci tensor, $R_{t \xi}=[\xi \dot{k}(t)] /[a(t) N(t)] \neq 0$, is a consequence of this fact. Hence, we were forced to introduce a midisuperspace model having a non-vanishing shift function $N_{\xi}(\xi, t)$. The metric was written as

$$
\begin{array}{r}
\mathrm{d} s^{2}=\left[-N^{2}(\xi, t)+\frac{N_{\xi}^{2}(\xi, t)}{a^{2}(\xi, t)}\right] \mathrm{d} t^{2}+2 N_{\xi}(\xi, t) \mathrm{d} \xi \mathrm{d} t \\
+a^{2}(\xi, t)\left[\mathrm{d} \xi^{2}+\sigma^{2}(\xi, t) \mathrm{d} \Omega^{2}(\theta, \varphi)\right] .
\end{array}
$$

The first step was to carry out the quantization of this midisuperspace model. Then, in a second step, we took into account in the quantum solutions the restrictions on the variables $a$ and $\sigma$ which, from Eq. (3), allows us to recover the metric (11). Consistency requires that we first treat the midisuperspace problem in order to come back to the minisuperspace model afterward. Correspondingly, the Wheeler-De Witt equation of the model remains a functional differential equation rendering the findings of general exact solutions problematic. In Ref. [4], only two particular semi-classical solutions were found for which the possibility of a topology change at the quantum level was explicitly demonstrated.

The present article aims at investigating the quantum behavior of the system described by the metric (11) by generalizing the midisuperspace description to a full superspace description in order to get more general conclusions than those reached in Ref. 4].

Quantities of great interest for this purpose are the Green functions since they completely characterize the quantum evolution of the system. For a point particle, the Green function $G\left(x_{\mathrm{f}}, t_{\mathrm{f}} ; x_{\mathrm{i}}, t_{\mathrm{i}}\right)$ represents the probability amplitude to find the particle at point $x_{\mathrm{f}}$ and time $t_{\mathrm{f}}$, knowing that it was at point $x_{\mathrm{i}}$ at the initial time $t_{\mathrm{i}}$. In fact, it is necessary to smooth out the Green function because, defined as before, it is not square integrable. Physically, this is due to the fact that the particle can never be localized exactly. In the present context, a Green function can also be defined and a similar interpretation can be made. In that case, the wave function depends on the volume of the spacelike sections $y \propto a^{3}$, on the three curvature $R$, and on a dust field $\chi(t)$ (rigorous definitions are given below), which plays the role of time: $\Psi=\Psi(y, R, \chi)$, see Ref. [6]. The Green function $G\left(y_{\mathrm{f}}, R_{\mathrm{f}}, \chi_{\mathrm{f}} ; y_{\mathrm{i}}, R_{\mathrm{i}}, \chi_{\mathrm{i}}\right)$ can now be defined as the probability amplitude of having a volume $y_{\mathrm{f}}$ and a threecurvature $R_{\mathrm{f}}$ at "time" $\chi_{\mathrm{f}}$ knowing that, at initial $\chi_{\mathrm{i}}$, the space volume was $y_{\mathrm{i}}$ and the three-curvature $R_{\mathrm{i}}$. Therefore, the Green function defined above represents the amplitude of probability for a topology transition as soon as $R_{\mathrm{f}}$ has not the same sign as $R_{\mathrm{i}}$. This is the quantity that we would like to calculate.

However, a full calculation of the Green functions is probably beyond our present computational capability and therefore, we are forced to rely on some approximation methods. Firstly, we will restrict ourselves to a semi-classical approximation of the Wheeler-De Witt equation. In this framework, the wave function will be given by the semi-classical wave function $\Psi=\exp (i S / \hbar)$. In the case of a topology change, the phase $S$ has a non-vanishing imaginary part, as in ordinary quantum tunneling, because topology change cannot be obtained classically, see Ref. [1]. Secondly, we will assume that the spatial sections of our Universe model are made of compact homogeneous backgrounds together with small perturbations around them. As we are interested in large scale quantum changes of topology in the homogeneous background and not on the small scale ones, typical of Wheeler's space-time foam, we will use the long-wavelength approximation, developed in Refs. 8, 9, 10, 11], which is valid whenever the characteristic scale of the problem is much bigger then the Hubble radius, and permits a complete analysis of the semi-classical wave function and Green functions in the full superspace. This will allows us to exhibit explicit analytical expressions for the Green functions. In particular, we will demonstrate that, for some transitions, the corresponding Green function vanishes, establishing thus selection rules for topology changes.

This article is organized as follows. In the second section, we briefly review the long wavelength approximation and the evaluation of the Hamilton-Jacobi function up to the second order (first order in the curvature). Then, in the third section, we perform the calculations in the case of real metrics and show that there is no quantum change of topology in this case. We also present a generalization of the results obtained in Ref. [10]. In the fourth section, we argue that complex metrics are necessary in order to obtain quantum changes of topology. We explicitly calculate the corresponding Green functions and discuss the main consequences that can be drawn from their expressions. In the last and fifth section we present our conclusions. 


\section{THE LONG WAVELENGTH APPROXIMATION FOR QUANTUM GRAVITY WITH DUST}

In this section, we discuss the long-wavelength approximation technique used to solve the HamiltonianJacobi equation which is nothing but the equation for the phase functional in a Wentzel-Kramer-Brillouin (WKB) semi-classical approximation to canonical quantum gravity. This technique consists in the expansion of the phase functional (or the generating functional in a Hamiltonian formulation of General Relativity) in a series of spatial gradients and was introduced by Salopek, Stewart and Parry [8, 9, 10] as a method for solving the full HamiltonJacobi equation for gravity interacting with matter. In Ref. 10] these authors were able to exhibit a recurrence formula from which they could obtain solutions to the Hamilton-Jacobi equation to any order of approximation for matter made of scalar and dust fields. This will be crucial for our treatment because we shall use the second order solution to obtain the Green functions associated with the WKB wave functional. As we will show, the solution in the second order approximation will allow us to describe changes of topology because the terms in the second order expansion of the WKB phase functional explicitly contain spatial curvature terms. As already mentioned, changes in the sign of these terms indicate a change of topology.

Let us now briefly describe the formalism introduced in Refs. [8, 9, 10]. The action for General Relativity interacting with a dust field $\chi$ is

$$
\begin{aligned}
S= & \int \mathrm{d}^{4} x \sqrt{-g}\left[\frac{1}{2 \kappa}{ }^{(4)} R-\frac{n}{2 m}\left(g^{\mu \nu} \partial_{\mu} \chi \partial_{\nu} \chi+m^{2}\right)\right. \\
& -V(\chi)]
\end{aligned}
$$

where $\kappa \equiv 8 \pi / m_{\mathrm{Pl}}^{2}, m_{\mathrm{Pl}}$ being the Planck mass. ${ }^{(4)} R$ is the Ricci scalar of the space-time metric $g_{\mu \nu}$, and $\chi$ is a velocity potential for irrotational dust particles with rest mass $m . V(\chi)$ is a potential for the dust field $\chi$ and $n$ is the rest number density of the dust particles. The dust field $\chi$ defines the four velocity field for dust particles by

$$
u^{\mu}=-g^{\mu \nu} \frac{1}{m} \chi_{, \nu}
$$

so that $\chi=$ const. will determine a congruence of spacelike hypersurfaces foliating the space-time (notice that $g^{\mu \nu} \chi_{, \mu} \chi_{, \nu}=-m^{2}$ ). Therefore, the dust field can be used as the time variable for our model, as well as in the Schrödinger-type equation obtained from the Wheeler-De Witt equation.

In the Arnowitt-Deser-Misner formalism the line element reads

$$
\mathrm{d} s^{2}=\left(-N^{2}+\gamma^{i j} N_{i} N_{j}\right) \mathrm{d} t^{2}+2 N_{i} \mathrm{~d} t \mathrm{~d} x^{i}+\gamma_{i j} \mathrm{~d} x^{i} \mathrm{~d} x^{i},
$$

where $N$ and $N_{i}$ are the lapse and shift functions, respectively, and $\gamma_{i j}$ is the three-metric of the spacelike hypersurface. Then, the action (4) can be rewritten as

$$
S=\int \mathrm{d}^{4} x\left(\pi^{\chi} \dot{\chi}+\pi^{i j} \dot{\gamma}_{i j}-N \mathcal{H}-N^{i} \mathcal{H}_{i}\right),
$$

where $\pi^{i j}$ are the momenta conjugate to $\gamma_{i j}$ and $\pi^{\chi}=$ $n \gamma^{1 / 2}\left(1+\chi, i \chi^{, i} / m^{2}\right)^{1 / 2}$ is the momentum conjugate to the dust field $\chi$. The quantities $\mathcal{H}, \mathcal{H}_{i}$ are given by the following expressions

$$
\begin{aligned}
\mathcal{H} & =\kappa \gamma^{-1 / 2} \pi^{i j} \pi^{k \ell}\left(2 \gamma_{j k} \gamma_{\ell i}-\gamma_{i j} \gamma_{k \ell}\right) \\
& -\frac{1}{2 \kappa} \gamma^{1 / 2} R+\left(m^{2}+\chi_{, i} \chi^{, i}\right)^{1 / 2} \pi^{\chi}+\gamma^{1 / 2} V(\chi),(8) \\
\mathcal{H}_{i} & =-2\left(\gamma_{i \ell} \pi^{\ell k}\right)_{, k}+\pi^{\ell k} \gamma_{\ell k, i}+\pi^{\chi} \chi_{, i},
\end{aligned}
$$

where $R$ is the Ricci scalar of the three-metric $\gamma_{i j}$.

At this point it is well worth making some dimensional analysis, in order to connect the conditions of validity of the long-wavelength approximation to the Hubble radius of the Universe. From Eq. (8), we can see that the longwavelength approximation implies that the first term on the right-hand side is much larger than the second term, at first order in the approximation. From the expression for $\pi^{i j}=\left(K^{i j}-h^{i j} K\right) / \kappa$, see Ref. [4], we can see that $\pi^{i j}$ has the dimension $L^{-3}$ (in the system of units where $\hbar=c=1$ ) where $L$ stands for a length. More explicitly,

$$
\pi^{i j} \sim \frac{1}{\ell_{\mathrm{Pl}}^{2} \ell_{\mathrm{H}}},
$$

where $\ell_{\mathrm{Pl}}=1 / m_{\mathrm{Pl}}$ is the Planck length and $\ell_{\mathrm{H}}$ is essentially the Hubble radius. Therefore, the validity of the long-wavelength approximation imposes that, see Eq. (8),

$$
\lambda \gg \ell_{\mathrm{H}},
$$

where the wavelength $\lambda$ is a typical length associated with the scales where spatial gradients are relevant. In other words, $\lambda$ must be greater than the characteristic Hubble radius of the model. In our scheme, topology change is a large-scale (global) phenomenon and the long-wavelength approximation is therefore the appropriate approximation to probe it. The above condition, obviously, should not contain the Planck length because Eq. (8) is a classical equation. Furthermore, in principle, there is no problem in applying this condition to closed models, even when their volumes becomes close to the Planck volume, (where, anyway, our semi-classical approximation should not be valid) if the Hubble radius at this epoch is smaller than the Planck length.

At the quantum level the above system may be quantized following the Dirac's prescription [12]. The superHamiltonian and super-momentum constraints given by Eqs. (8) and (9) become operators and, when applied to the wave functional of the system, result in two relations which express that only a restricted region of the Hilbert space of wave functionals contains the physical states of the theory:

$$
\mathcal{H} \Psi=0, \quad \mathcal{H}_{i} \Psi=0 .
$$


The first equation is the well-known Wheeler-De Witt equation whereas the second one is the so-called quantum momentum constraint. In what follows, our treatment of quantum changes of topology will be done at the level of the semi-classical approximation. This means that the wave functionals are assumed to be of the WKB form, namely

$$
\Psi=\mathrm{e}^{i S / \hbar}
$$

where $S$ is the action. At order $\hbar^{0}$, Eqs. (8) and (9) reduce to the Hamilton-Jacobi functional equation

$\mathcal{H}(x)=\gamma^{-1 / 2} \kappa \frac{\delta S}{\delta \gamma_{i j}(x)} \frac{\delta S}{\delta \gamma_{k \ell}(x)}\left[2 \gamma_{i \ell}(x) \gamma_{j k}(x)-\gamma_{i j}(x) \gamma_{k \ell}(x)\right]+\sqrt{m^{2}+\gamma^{i j} \chi_{, i} \chi, j} \frac{\delta S}{\delta \chi(x)}+\gamma^{1 / 2} V(\chi)-\frac{1}{2 \kappa} \gamma^{1 / 2} R=0$

and to the momentum constraint equation

$\mathcal{H}_{i}(x)=-2\left[\gamma_{i k} \frac{\delta S}{\delta \gamma_{k j}(x)}\right]_{, j}+\frac{\delta S}{\delta \gamma_{\ell k}(x)} \gamma_{\ell k, i}+\frac{\delta S}{\delta \chi} \chi_{, j}=0$

the solution of which is the phase $S$ of the WKB wave functional, see Eq. [11). In Ref. [10], Parry, Salopek and Stewart have been able to derive solutions for these equations using the long wavelength approximation. The method is based on the so-called spatial gradient expansion of the phase functional $S$. It consists in expanding $S$ in a series of terms according to the number of spatial gradients they contain, namely $S=S^{(0)}+S^{(2)}+S^{(4)}+\cdots$. As a consequence, the Hamilton-Jacobi equation can be grouped in terms with an even number of spatial derivatives $\mathcal{H}=\mathcal{H}^{(0)}+\mathcal{H}^{(2)}+\mathcal{H}^{(4)}+\cdots$. At zeroth order, the Hamilton-Jacobi equation reduces to

$$
\begin{aligned}
\mathcal{H}^{(0)}= & \gamma^{-1 / 2} \kappa \frac{\delta S^{(0)}}{\delta \gamma_{i j}} \frac{\delta S^{(0)}}{\delta \gamma_{k \ell}}\left(2 \gamma_{j k} \gamma_{\ell i}-\gamma_{i j} \gamma_{k \ell}\right) \\
& +m \frac{\delta S^{(0)}}{\delta \chi}+\gamma^{1 / 2} V(\chi)=0 .
\end{aligned}
$$

For a dust field $\chi$ with a vanishing potential, $V(\chi)=0$, the invariance under diffeomorphisms of the generating functional suggests a solution of the form

$$
S^{(0)}=-\frac{2}{\kappa} \int \mathrm{d}^{3} x \gamma^{1 / 2} H(\chi) .
$$

This functional satisfies the Hamilton-Jacobi relation if $H(\chi)$ is a solution of $H^{2}=-(2 m / 3) \partial H / \partial \chi$ yielding

$$
H(\chi)=\frac{2 m}{3(\chi-\tilde{\chi})}
$$

where $\tilde{\chi}$ is an integration constant. The functional $S^{(0)}$ is clearly invariant under coordinate transformations and, therefore, satisfies Eq. (13). In addition, it contains no spatial derivatives. For the class of generating functionals given by Eq. (15), the second-order Hamilton-Jacobi equation reads

$$
\begin{aligned}
\mathcal{H}^{(2)}= & 2 H \gamma_{i j} \frac{\delta S^{(2)}}{\delta \gamma_{i j}}+m \frac{\delta S^{(2)}}{\delta \chi}+\frac{1}{2 m} \gamma^{i j} \chi_{, i} \chi, j \frac{\delta S^{(0)}}{\delta \chi} \\
& -\frac{1}{2 \kappa} \gamma^{1 / 2} R=0
\end{aligned}
$$

In Ref. [10], Parry, Salopek and Stewart obtained a diffeomorphisms invariant solution for $S^{(2)}$ which can be written as

$$
S^{(2)}=\frac{1}{\kappa} \int \mathrm{d}^{3} x \gamma^{1 / 2} J(\chi) R,
$$

where the function $J(\chi)$ is obtained by substituting Eq. (18) into Eq. (17) and using the zeroth order solution (15). This leads to

$$
H J+m \frac{\partial J}{\partial \chi}=\frac{1}{2}
$$

which yields for $S^{(2)}$

$$
S^{(2)}=\frac{1}{\kappa} \int \mathrm{d}^{3} x \gamma^{1 / 2}\left[\frac{3}{10 m}(\chi-\tilde{\chi})+D(\chi-\tilde{\chi})^{-2 / 3}\right] R,
$$

where $D$ is an arbitrary integration constant. We notice the fact that $S^{(2)}$ contains the three-curvature term $R$ whereas this is not the case for $S^{(0)}$. Since a topology change is signaled by a change of sign of the threecurvature $R$, it is necessary to push the long wavelength expansion to the second order in order to have non trivial effects. In other words, the quantum topology changes can only be studied at this (second) order.

\section{THE GREEN FUNCTION WITH LORENTZIAN METRICS}

\section{A. General Derivation}

Let us now consider the semi-classical wave function up to the second order in the long wavelength approximation $\Psi=\exp \left\{i\left[S^{(0)}+S^{(2)}\right] / \hbar\right\}$ with $S^{(0)}$ and $S^{(2)}$ given by Eqs. (15) and (20), as explained in the previous section. We restrict ourselves to geometries of the form given in Eq. (11). Then, the integrals in Eqs. (15) and (20) can be performed exactly yielding

$$
\begin{aligned}
\Psi(y, R, \chi)= & \exp \left\{\frac { i } { \hbar \kappa } \left[-2 y H+\frac{3 y R(\chi-\tilde{\chi})}{10 m}\right.\right. \\
& \left.\left.+\frac{y R D}{(\chi-\tilde{\chi})^{2 / 3}}\right]\right\},
\end{aligned}
$$


where the quantity $y$ given by

$$
y \equiv \int \mathrm{d}^{3} x \gamma^{1 / 2}=V a^{3}
$$

is the total volume associated with the metric (1). $V$ is the constant volume of the cell corresponding to the homogeneous polyhedron of the tessellation of the spatial sections that are compactified. As previously, $H$ can be expressed as $H=2 \mathrm{~m} /[3(\chi-\tilde{\chi})]$ and $R$ is the threecurvature which can be written as $R=6 \mathcal{K} / a^{2}$. The Green functions $G$ are defined by

$$
\begin{aligned}
\Psi(y, R, \chi) \equiv & \int_{0}^{\infty} \mathrm{d} y^{\prime} \int_{-\infty}^{\infty} \mathrm{d} R^{\prime} G\left(y, R, \chi ; y^{\prime}, R^{\prime}, \chi^{\prime}\right) \\
& \times \Psi\left(y^{\prime}, R^{\prime}, \chi^{\prime}\right) .
\end{aligned}
$$

Our goal is now to establish the expression for the Green function itself from the above equation. For this purpose, it is convenient to use a new variable $v$, which is a combination of $y$ and $R$, namely $v \equiv y R$. To obtain the Green function, we use the fact that Eq. (23) is valid for any value of the integration constants $\tilde{\chi}$ and $D$. However, instead of the integration constants $\tilde{\chi}$ and $D$, it will be more appropriate to work with new integration constants defined by the following relations (for fixed $\chi^{\prime}$ )

$$
\begin{aligned}
H^{\prime}(\tilde{\chi}) & \equiv \frac{2 m}{3\left(\chi^{\prime}-\tilde{\chi}\right)} \\
J^{\prime}(\tilde{\chi}, D) & \equiv \frac{3}{10 m}\left(\chi^{\prime}-\tilde{\chi}\right)+D\left(\chi^{\prime}-\tilde{\chi}\right)^{-2 / 3} .
\end{aligned}
$$

We have just replaced the two constants $(\tilde{\chi}, D)$ by two new constants $\left(H^{\prime}, J^{\prime}\right)$. Let us notice that $J^{\prime}$ is in fact $J\left(\chi^{\prime}\right)$, see Eqs. (18) and (20), hence its name. If we inverse the above relations, one arrives at

$$
\begin{aligned}
\tilde{\chi}\left(H^{\prime}\right) & =\chi^{\prime}-\frac{2 m}{3 H^{\prime}} \\
D\left(H^{\prime}, J^{\prime}\right) & =J^{\prime}\left(\frac{2 m}{3 H^{\prime}}\right)^{2 / 3}-\frac{3}{10 m}\left(\frac{2 m}{3 H^{\prime}}\right)^{5 / 3}
\end{aligned}
$$

Let us stress again that these expressions are defined for a fixed value of $\chi^{\prime}$. With these new definitions and the explicit expression of the wave function given in Eq. (21), Eq. (23) now reads

$$
\begin{aligned}
\exp \left(\frac { i } { \hbar \kappa } \left\{-2 y H+\frac{3 v\left[\chi-\tilde{\chi}\left(H^{\prime}\right)\right]}{10 m}+\frac{v D\left(H^{\prime}, J^{\prime}\right)}{\left.\left.\left[\chi-\tilde{\chi}\left(H^{\prime}\right)\right]^{2 / 3}\right\}\right)=}\right.\right. & \int_{0}^{\infty} \frac{\mathrm{d} y^{\prime}}{y^{\prime}} \int_{-\infty}^{\infty} \mathrm{d} v^{\prime} G\left(y, \frac{v}{y}, \chi ; y^{\prime}, \frac{v^{\prime}}{y^{\prime}}, \chi^{\prime}\right) \\
& \times \exp \left[\frac{i}{\hbar \kappa}\left(-2 y^{\prime} H^{\prime}+v^{\prime} J^{\prime}\right)\right]
\end{aligned}
$$

We see the effect of having introduced the "new" integration constants: the left-hand-side of the previous equation now depends on both $\chi$ and $\chi^{\prime}$. In order to extract $G\left(y, v / y, \chi ; y^{\prime}, v^{\prime} / y^{\prime}, \chi^{\prime}\right)$, we multiply the previous equation by the factor

$$
\exp \left[\frac{i}{\hbar \kappa}\left(2 y^{\prime \prime} H^{\prime}-v^{\prime \prime} J^{\prime}\right)\right]
$$

and integrate it over $J^{\prime}$ and $H^{\prime}$ from $-\infty$ to $\infty$. The result of this integration on the right-hand-side of Eq. (28) is very simple, yielding two Dirac delta functions whose arguments are $v^{\prime}-v^{\prime \prime}$ and $y^{\prime}-y^{\prime \prime}$. This allows us to perform the integration over $v^{\prime}$ and $y^{\prime}$ immediately. The result reads

$$
\begin{aligned}
& G\left(y, \frac{v}{y}, \chi ; y^{\prime \prime}, \frac{v^{\prime \prime}}{y^{\prime \prime}}, \chi^{\prime}\right) \\
= & \frac{y^{\prime \prime}}{2 \pi^{2} \hbar^{2} \kappa^{2}} \int_{-\infty}^{\infty} \mathrm{d} H^{\prime} \int_{-\infty}^{\infty} \mathrm{d} J^{\prime} \exp \left\{\frac{i}{\hbar \kappa}\left[-\frac{2 y b H^{\prime}}{H^{\prime}+b}+2 y^{\prime \prime} H^{\prime}-v^{\prime \prime} J^{\prime}+v\left(J^{\prime}-\frac{1}{5 H^{\prime}}\right)\left(\frac{b}{H^{\prime}+b}\right)^{2 / 3}+\frac{v}{5}\left(\frac{1}{b}+\frac{1}{H^{\prime}}\right)\right]\right\}
\end{aligned}
$$

where $b \equiv 2 m /\left[3\left(\chi-\chi^{\prime}\right)\right]$. The first term in the argument of the exponential has been obtained by using the relation
$H=2 m /[3(\chi-\tilde{\chi})]$ and then Eqs. (26) and (27). The two last terms have been obtained by using the explicit 
expressions of $\tilde{\chi}$ and $D$, see Eqs. (26) and (27). The above equation is the main result of this section. Clearly, the central issue is now the calculation of the integrals over $J^{\prime}$ and $H^{\prime}$. From Eq. (30), we see that the integral over $H^{\prime}$ is to be performed along the real axis. However, in the next subsection, dealing with the case of flat hypersurfaces, the integration over $H^{\prime}$ will be performed in the complex plane, along a closed contour. It is important to notice that this procedure is just a technical trick, allowing us to simplify the calculation of the integral by the use of the Cauchy theorem, but it has no deep physical meaning. On the contrary, in section IV, the integration in the complex plane has a physical meaning and, as we will argue, is in fact necessary. Of course, all the following complex contours turn out to be, after having taken the appropriate limits, equivalent to the integration along the real axis as given in Eq. (30).

\section{B. The Case of Flat Hypersurfaces}

In this section we treat the case where either the starting hypersurface or the resulting hypersurface (after the topology change) is flat.

Let us first start with the case $R=0$ and $R^{\prime \prime} \neq 0$, hence $v=0$ but $v^{\prime \prime} \neq 0$. In this case, the integration over $J^{\prime}$ is very simple and yields the Dirac function $\delta\left(v^{\prime \prime}\right)$. Consequently, in order to obtain a non-vanishing Green function, one must take $v^{\prime \prime}=0$, that is to say $R^{\prime \prime}=0$. This means that the three-curvature remains the same (i.e. zero) and there is obviously no topology change. At this point, however, we go further and depict the calculation of the Green function despite the fact that there is no change of topology and despite the fact that the final result is already known, see Ref. 11]. The main reason for doing this is that this will exemplify techniques that will be used in this paper later on. This calculation is therefore a warm-up. Eq. (30) now reduces to

$$
\begin{aligned}
& G\left(y, 0, \chi ; y^{\prime \prime}, 0, \chi^{\prime}\right) \\
= & \frac{y^{\prime \prime}}{2 \pi^{2} \hbar^{2} \kappa^{2}} \int_{-\infty}^{\infty} \mathrm{d} H^{\prime} \exp \left[\frac{i}{\hbar \kappa}\left(\frac{2 y b H^{\prime}}{H^{\prime}+b}+2 y^{\prime \prime} H^{\prime}\right)\right] .
\end{aligned}
$$

As mentioned before, the integral in the right-hand-side of Eq. (31) can be calculated by extending $H^{\prime}$ to the complex plane with the choice of the integration contour represented by the solid line in Fig. 1] This contour contains the pole $H^{\prime}=-b$ of the exponential function appearing in the right-hand-side of Eq. (31). The fact that $H^{\prime}=-b$, which is a pole of the argument of the exponential, is also a pole of the exponential itself can easily be seen if one uses the Taylor expansion of the exponential function. When the limits $\rho \rightarrow+\infty$ and $\epsilon \rightarrow 0$ are taken, see Fig. 11 the integral over $H^{\prime}$ in the right-hand-side of Eq. (31) reduces to an integration on the real axis, the contribution coming from the big semicircle in the upper-half complex plane going to zero as a consequence

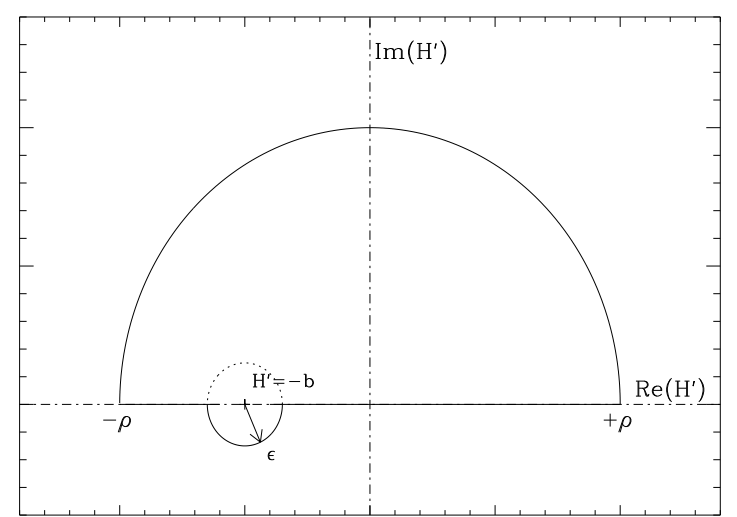

FIG. 1: The contour of integration $\mathcal{C}$ in the complex plane $\left[\Re\left(H^{\prime}\right), \Im\left(H^{\prime}\right)\right]$. The contour chosen in this article is the solid line contour. Because of the Cauchy's theorem, $\mathcal{C}$ is equivalent to the small circle contour centered at $H^{\prime}=-b$.

of the Jordan's lemma. Because of the Cauchy's theorem, the integration along $\mathcal{C}$ is in fact equivalent to the integration over a small circle centered around $H^{\prime}=-b$, see Fig. 11 This was the contour considered in Ref. 11] which is therefore equivalent to the contour $\mathcal{C}$ used in this article. As a consequence, one obtains

$$
\begin{gathered}
G\left(y, 0, \chi ; y^{\prime \prime}, 0, \chi^{\prime}\right)=\frac{y^{\prime \prime}}{2 \pi^{2} \hbar^{2} \kappa^{2}} \\
\times \int_{\mathcal{C}} \mathrm{d} H^{\prime} \exp \left[\frac{i}{\hbar \kappa}\left(-\frac{2 y b H^{\prime}}{H^{\prime}+b}+2 y^{\prime \prime} H^{\prime}\right)\right] .
\end{gathered}
$$

It has been shown in Ref. [1] that the integration over $H^{\prime}$ along the contour $\mathcal{C}$ can then be done explicitly. The exact result is expressed in Eq. (4.25) of Ref. [1] in terms of a Bessel function of order one. We do not present this result here since this aspect of the calculation has been given in details in Ref. [11].

Let us now consider the other possibility where $R \neq 0$, hence $v \neq 0$ but now $R^{\prime \prime}=0\left(v^{\prime \prime}=0\right)$. Despite the fact that the argument of the exponential function is now more complicated, the integration over $J^{\prime}$ can still be performed very easily and yields the Dirac delta function

$$
\delta\left[v\left(\frac{b}{H^{\prime}+b}\right)^{2 / 3}\right]
$$

Since the argument of the Dirac function has no zeros in $H^{\prime}$, the integration over $H^{\prime}$ leads to a vanishing Green function.

Hence, we have established the following result: there is no quantum change of topology from or to a flat spacelike hypersurface in the homogeneous background, at least at second order in the long-wavelength approximation. 


\section{The Case of Curved Hypersurfaces}

We now assume that both $R$ and $R^{\prime}$, and hence $v$ and $v^{\prime \prime}$, do not vanish. This time the integration over $J^{\prime}$, in the right-hand-side of Eq. (30), gives the following Dirac function [to be compared with Eq. (33)]

$$
\delta\left[v\left(\frac{b}{H^{\prime}+b}\right)^{2 / 3}-v^{\prime \prime}\right] .
$$

Since $H^{\prime}$ is real, the argument of the Dirac function has zeros if and only if $R$ and $R^{\prime}$ (or, equivalently, $v$ and $v^{\prime \prime}$ ) have the same sign and, in this case, the zeros are given by

$$
H^{\prime}=H_{0}^{\prime \mp} \equiv-b\left[1 \mp\left(\frac{v}{v^{\prime \prime}}\right)^{3 / 2}\right] .
$$

If $R$ and $R^{\prime}$ have not the same sign, the corresponding Green function is zero and quantum topology change is forbidden. Therefore, as in the flat case, there is no quantum change of topology.

Despite this fact, and for the sake of completeness, we now present the resulting Green function since this represents a non trivial generalization of the results obtained in Ref. [11]. Let us notice that this case is different from the one given in Eq. (31). There, we had a Dirac function of $v^{\prime \prime}$ only and, therefore, it was possible to put the Dirac function outside the integral over $H^{\prime}$. Here, we face a different situation since, this time, the Dirac function does depend on $H^{\prime}$ (the second flat case, i.e. $v \neq 0$, also presented this feature but since the argument of the Dirac function had no zeros, this case was in fact trivial). In order to calculate the integral (30) for the present case, we express the Dirac function as

$$
\begin{aligned}
& \delta\left[v\left(\frac{b}{H^{\prime}+b}\right)^{2 / 3}-v^{\prime \prime}\right]=\frac{3 b}{2|v|}\left(\frac{v^{\prime \prime}}{v}\right)^{-5 / 2} \\
& \times\left[\delta\left(H^{\prime}-H_{0}^{\prime-}\right)+\delta\left(H^{\prime}-H_{0}^{\prime+}\right)\right]
\end{aligned}
$$

and the integration becomes trivial. As a consequence, the Green function is given by the sum of two contributions coming from the two Dirac functions in Eq. (36)

$$
G_{r}=\left(G_{+}+G_{-}\right) \Theta\left(\frac{\mathcal{K}}{\mathcal{K}^{\prime \prime}}\right)
$$

where $\Theta$ is the step function and where $G_{ \pm}$are given by

$$
\begin{aligned}
& G_{+}=\frac{3 m y^{\prime \prime} x^{5 / 2}}{\pi \hbar \kappa|v|\left(\chi-\chi^{\prime \prime}\right)} \exp \left\{\frac{i}{\hbar \kappa}\left[-\frac{2 y b\left(x^{3 / 2}-1\right)}{x^{3 / 2}}+2 y^{\prime \prime} b\left(x^{3 / 2}-1\right)+\frac{\mathcal{K} y^{1 / 3}\left(x^{5 / 2}-1\right)}{5 b\left(x^{3 / 2}-1\right) x}\right]\right\}, \\
& G_{-}=\frac{3 m y^{\prime \prime} x^{5 / 2}}{\pi \hbar \kappa|v|\left(\chi-\chi^{\prime \prime}\right)} \exp \left\{\frac{i}{\hbar \kappa}\left[-\frac{2 y b\left(x^{3 / 2}+1\right)}{x^{3 / 2}}-2 y^{\prime \prime} b\left(x^{3 / 2}+1\right)+\frac{\mathcal{K} y^{1 / 3}\left(x^{5 / 2}+1\right)}{5 b\left(x^{3 / 2}+1\right) x}\right]\right\},
\end{aligned}
$$

with $x \equiv\left(y / y^{\prime \prime}\right)^{1 / 3}$. This completes our study of the Green functions in the case of real metrics. We have demonstrated that there is no quantum change of topology in that case, as long as this transition involves a change of sign in the curvature of the spatial sections. Our formulation does not allow to signal a change of topology when the transition involves compactified spacelike sections with distinct topology but having the same sign of curvature, a fact that would require a completely distinct and much more elaborated approach to the problem. These conclusions are valid up to second order in the gradient expansion.

\section{THE GREEN FUNCTION WITH COMPLEX METRICS}

We have just seen in the previous section that there is no change of topology if $H^{\prime}$ remains real. This is linked to the fact that the equation $v^{\prime \prime}=v\left[b /\left(H^{\prime}+b\right)\right]^{2 / 3}$ has no solution on the real axis of $H^{\prime}$ if $v$ and $v^{\prime \prime}$ have opposite signs. It is worth recalling again that the extension of $H^{\prime}$ to the complex plane made in section III, in the case of flat hypersurfaces, was a mere mathematical trick to calculate the integrals, albeit all the poles inside the contours were located on the real axis. On the other hand, the above equation possesses a solution in the complex plane, namely

$$
H^{\prime}=H_{0}^{\prime} \equiv-b\left(1+i\left|\frac{v}{v^{\prime \prime}}\right|^{3 / 2}\right)
$$

and, therefore, this strongly suggests to consider $H^{\prime}$ as a true complex variable. As we will discuss in the present section, the complexification of $H^{\prime}$, with its natural consequence namely a complexification of the metric itself, is a physical requirement. This will lead us to interpret topology change as a quantum tunneling effect. In the case of complex metrics, the corresponding Green function needs to be modified and its expression can be obtained from the expressions derived before. We now describe how this can be done. 


\section{A. General derivation}

Changes of topology described by the metric (1) requires a complicated midisuperspace formulation of the problem, as described in the Introduction and explained in more details in Ref. [4]. However, in the case of curved spatial hypersurfaces, the midisuperspace formulation can be circumvented and the problem greatly simplified by the introduction of complex metrics. Let us now define the metric according to

$\mathrm{d} s^{2}=-N_{\mathrm{c}}^{2}(t) \mathrm{d} t^{2}+a^{2}(t)\left[\mathrm{d} z^{2}+\sin ^{2} z\left(\mathrm{~d} \theta^{2}+\sin ^{2} \theta \mathrm{d} \varphi^{2}\right)\right]$

and allow $N_{\mathrm{c}}$ and $z$ to be complex. Setting $N_{\mathrm{c}}=N$ and $z=\xi$ to be real yields, let us say for the final configuration, a positively curved spatial hypersurface in a Lorentzian four-geometry, while requiring initially that $N_{\mathrm{c}}=-i N$ and $z=-i \xi$ are purely imaginary yields a negatively curved spatial hypersurface, also in a Lorentzian four-geometry [of course, one can interchange the transition by choosing sinh instead of sin in Eq. (41)]. Therefore, the transition from positively to negatively curved spatial sections may be obtained with a mere complexification of the metric, without changing the functional form of the metric in Eq. (41) or without needing to introduce a time dependence on $\mathcal{K}$, as in Eq. (11). In other words, the transition described above can be actually realized by following trajectories in the space of complex metrics. Notice also that, with the complexification procedure, there is an overall change of sign in the 4-metric while the transition from the initial to the final configuration occurs, implying a passage through degenerate metrics. This is typical of transitions with a change of topology as pointed out in Ref. [13]. Let us also emphasize that the use of complex trajectories in order to describe the tunneling effect is standard and has been used elsewhere, for instance in the study of chaotic potentials or in the calculation of the rotational spectra of molecules, see Ref. [14].

The change in the lapse function, $N_{\mathrm{c}}=-i N$, is equivalent to a Wick rotation to the imaginary time $\tau=-i t$, if one understands $t$ as the proper time of observers whose trajectories are orthogonal to the spacelike hypersurfaces. In this context, one needs to adapt the ADM formalism presented at the beginning of this article in order to obtain the Green function that will describe a topology change. We now turn to this question.

The Euclidean action defined as $I \equiv-i S\left[g_{\mathrm{E}}\right]$, see Ref. [13], is given by

$$
\begin{aligned}
I= & -\int \mathrm{d}^{4} x \sqrt{g_{\mathrm{E}}}\left[\frac{1}{2 \kappa}{ }^{(4)} R\left(g_{\mathrm{E}}\right)\right. \\
& \left.-\frac{n}{2 m}\left(g_{\mathrm{E}}^{\mu \nu} \partial_{\mu} \chi \partial_{\nu} \chi+m^{2}\right)-V(\chi)\right],
\end{aligned}
$$

where $g_{\mathrm{E}}$ denotes the Euclidean 4-geometry whose line element can be expressed as

$$
\mathrm{d} s^{2}=\left(N^{2}+\gamma^{i j} N_{i} N_{j}\right) \mathrm{d} t^{2}+2 N_{i} \mathrm{~d} t \mathrm{~d} x^{i}+\gamma_{i j} \mathrm{~d} x^{i} \mathrm{~d} x^{i}
$$

Note that we have used the relation $\sqrt{-g_{\mathrm{c}}}=-i \sqrt{g_{\mathrm{E}}}$, where $g_{\mathrm{c}}$ is a Lorentzian metric complexified as in Eq. (41). Having determined what the action is, we can study the Hamiltonian formalism. Calculations very similar to those performed in the standard ADM formalism show that the Hamiltonian obtained from the Euclidean action given above reads

$$
H_{\mathrm{E}}=N \mathcal{H}_{\mathrm{E}}+N^{i} \mathcal{H}_{i}
$$

where $\mathcal{H}_{\mathrm{E}}$ and $\mathcal{H}_{i}$ are respectively given by

$$
\begin{aligned}
\mathcal{H}_{\mathrm{E}}= & -\kappa \gamma^{-1 / 2} \pi^{i j} \pi^{k \ell}\left(2 \gamma_{j k} \gamma_{\ell i}-\gamma_{i j} \gamma_{k \ell}\right)-\frac{1}{2 \kappa} \gamma^{1 / 2} R \\
& +i\left(m^{2}+\chi{ }_{, j} \chi^{, j}\right)^{1 / 2} \pi^{\chi}+\gamma^{1 / 2} V(\chi) \\
\mathcal{H}_{i}= & -2\left(\gamma_{i \ell} \pi^{\ell k}\right)_{, k}+\pi^{\ell k} \gamma_{\ell k, i}+\pi^{\chi} \chi_{, i}
\end{aligned}
$$

and where, as before, $\pi^{i j}$ are the conjugate momenta to $\gamma_{i j}$ and $\pi^{\chi}$ is the conjugate momentum to the dust field $\chi$. The quantity $R$ is the Ricci scalar of the threemetric $\gamma_{i j}$. From the expression of $\mathcal{H}_{\mathrm{E}}$ derived above, one can establish the Euclidean Hamilton-Jacobi equation. It reads

$$
-\gamma^{-1 / 2} \kappa \frac{\delta S}{\delta \gamma_{i j}(x)} \frac{\delta S}{\delta \gamma_{k \ell}(x)}\left[2 \gamma_{i \ell}(x) \gamma_{j k}(x)-\gamma_{i j}(x) \gamma_{k \ell}(x)\right]+i \sqrt{m^{2}+\gamma^{i j} \chi_{, i} \chi, j} \frac{\delta S}{\delta \chi(x)}+\gamma^{1 / 2} V(\chi)-\frac{1}{2 \kappa} \gamma^{1 / 2} R=0(, 47)
$$

while the momentum constraint equation remains the same as in Eq. (13). Comparing Eq. (47) with Eq. (12), one can see that going to the imaginary time (by means of the Wick rotation) changes the sign of the kinetic term in the gravitational sector. This is a well-known modification when going from Lorentzian to Euclidean signatures. On the other hand, the matter sector, containing the term $\delta S / \delta \chi$, is multiplied by complex number $i$. This latter change was also expected since $\chi$ plays the role of time. Setting $\tau=-i t$ implies $\chi_{\mathrm{c}}=-i \chi$ after the Wick rotation, hence the above result.

Having the Euclidean Hamilton-Jacobi equation and the momentum equation at our disposal, we now seek solutions using the gradient expansion. In the following, 
quantities with the index c refer to the metric (41) after the Wick rotation $\left(N_{\mathrm{c}}=-i N, z\right.$ complex $)$ while quantities without index refer to the (real) Lorentzian metric (41) $(N=$ real, $\xi$ real). As it was the case before, see Eq. (15), the invariance by reparametrization suggests a solution of the form

$$
S_{\mathrm{c}}^{(0)}=-\frac{2}{\kappa} \int \mathrm{d}^{3} x \gamma_{\mathrm{c}}^{1 / 2} H_{\mathrm{c}}(\chi) .
$$

Inserting this form into the Euclidean Hamilton-Jacobi equation yields, at the zero order, $H_{\mathrm{c}}^{2}=(2 \mathrm{im} / 3) \partial H_{\mathrm{c}} / \partial \chi$. This equation should be compared with the equation written after Eq. (15). The difference between those two equations is a factor $-i$ as expected. The corresponding solution can be written as $H_{\mathrm{c}}=-i H$, where $H(\chi)=2 m /[3(\chi-\tilde{\chi})]$. Again, this solution bears some close resemblance with the solution of Eq. (16).

At second order, the diffeomorphism invariant solution reads

$$
S_{\mathrm{c}}^{(2)}=\frac{1}{\kappa} \int \mathrm{d}^{3} x \gamma_{\mathrm{c}}^{1 / 2} J_{\mathrm{c}}(\chi) R_{\mathrm{c}},
$$

and is similar to the solution of Eq. (20). Applying the same method as before, i.e. inserting the above equation into the Euclidean Hamilton-Jacobi equation, yields the following relation

$$
-H_{\mathrm{c}} J_{\mathrm{c}}+i m \frac{\partial J_{\mathrm{c}}}{\partial \chi}=i H J_{\mathrm{c}}+i m \frac{\partial J_{\mathrm{c}}}{\partial \chi}=\frac{1}{2} .
$$

Since we have already established the expression of $H_{\mathrm{c}}$, the above relation is a differential equation for the quantity $J_{\mathrm{c}}$ only. The solution is very similar to the one found in section II of this paper. It reads $J_{\mathrm{c}}=-i J$, where, as can be deduced from Eq. (20), one has

$$
J(\tilde{\chi}, D)=\frac{3}{10 m}(\chi-\tilde{\chi})+D(\chi-\tilde{\chi})^{-2 / 3} .
$$

We now have at our disposal the Euclidean action at zeroth and second order in full generality, i.e. for any metric tensor. As before, we now restrict our considerations to the metrics of the form (41). This allows us to perform the integrals (48) and (49) exactly. However, in the Euclidean case, one has to pay attention to the following fact. When the transformation $z=-i \xi$ is made, the three-metric gets modified by an overall sign (besides the change from sin to sinh). This implies that $\gamma_{\mathrm{c}}^{1 / 2}=-i \gamma^{1 / 2}$ and, hence, the volume $y_{\mathrm{c}}$ can be written as

$$
y_{\mathrm{c}} \equiv \int \mathrm{d}^{3} x \gamma_{\mathrm{c}}^{1 / 2}=-i y
$$

For the same reason, the three-curvature satisfies $R_{\mathrm{c}} \equiv$ $R\left(\gamma_{\mathrm{c}}^{\mu \nu}\right)=-R\left(\gamma^{\mu \nu}\right) \equiv-R$. Combining the previous results, one has $v_{\mathrm{c}}=y_{\mathrm{c}} R_{\mathrm{c}}=i y R=i v$.

We are now in a position where we can calculate the semi-classical wave function in the long wavelength approximation at the time $\chi$. The result reads

$$
\begin{aligned}
\Psi\left(y_{\mathrm{c}}, R_{\mathrm{c}}, \chi\right) & =\exp \left[\frac{i}{\hbar \kappa}\left(-2 y_{\mathrm{c}} H_{\mathrm{c}}+v_{\mathrm{c}} J_{\mathrm{c}}\right)\right] \\
& =\exp \left[\frac{i}{\hbar \kappa}(2 y H+v J)\right]
\end{aligned}
$$

This solution should be compared with Eq. (21). One notices that there is a difference, namely a change of sign in the first term of the exponential. As we are going to show below, this simple change of sign turns out to be crucial in order to obtain a Green function which allows a quantum change of topology.

The last step is to calculate the Green function explicitly. This Green function describes a transition from a situation where the wave function is expressed by the standard formula, Eq. (21), to a case where the wave function is modified by the above-mentioned change of sign, see Eq. (53) (or vice versa if one wishes to consider the opposite transition). As a consequence, in order to obtain the Green function, we just need to repeat the calculations performed between Eq. (23) and Eq. (32), bearing in mind that, in the final (or initial) configuration, the wave function is now described by Eq. (53). We obtain the following result

$$
G_{i}\left(y, R, \chi ; y^{\prime}, R^{\prime}, \chi^{\prime}\right)=\frac{y^{\prime}}{2 \pi^{2} \hbar^{2} \kappa^{2}} \int_{\mathcal{C}} \mathrm{d} H^{\prime} \int_{-\infty}^{\infty} \mathrm{d} J^{\prime} \exp \left\{\frac{i}{\hbar \kappa}\left[-2 y H-2 y^{\prime} H^{\prime}-v^{\prime} J^{\prime}+\frac{3 v(\chi-\tilde{\chi})}{10 m}+\frac{v D}{(\chi-\tilde{\chi})^{2 / 3}}\right]\right\}
$$

At this point, one should compare the above result with Eq. (32). Being given the previous considerations, it does not come as a surprise that the sign of the second term in the exponential is different. The origin of the difference is clearly the modified wave function of Eq. (53). Apart from this (crucial) difference, the previous expression is identical to Eq. (30). Let us also notice that, in order to derive the Green function, we have already assumed that the contour $\mathcal{C}$ reduces to an integration along the real axis. This allows us to extract the Green function directly. We will show below that this is indeed the case. 


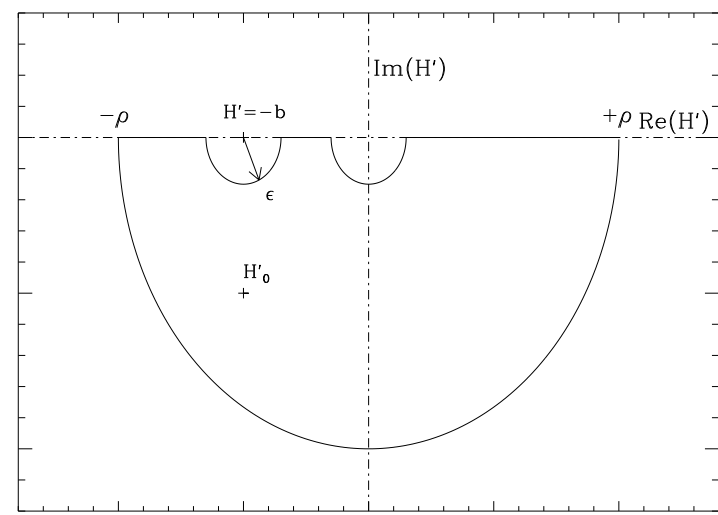

FIG. 2: The contour of integration $\mathcal{C}$ in the complex plane $\left[\Re\left(H^{\prime}\right), \Im\left(H^{\prime}\right)\right]$ in the case of curved hypersurfaces for the modified Green function. The contour chosen is the solid line contour which contains the complex pole of the Dirac function $H_{0}^{\prime}$ but avoids the poles of the exponential function, namely $H^{\prime}=0$ and $H^{\prime}=-b$.

\section{B. Explicit calculation of the Green function}

Equipped with the modified Green function (55), we now return to our main subject, i.e the study of the quan- tum topology changes. We now consider the case where $v$ and $v^{\prime}$ have opposite signs, namely $\operatorname{sign}(v)=-\operatorname{sign}\left(v^{\prime}\right)$.

The first step is to perform the integration over $J^{\prime}$. This yields the Dirac $\delta$-function of Eq. (34) (except that one should replace $v^{\prime \prime}$ by $v^{\prime}$ ). This function has the root given in Eq. (40), i.e. $H^{\prime}=H_{0}^{\prime}$. The second step is the choice of the contour $\mathcal{C}$. In the complex plane, integration of a Dirac $\delta$-function can be represented by an integration along a small circle around the roots of its argument. For this reason, the integration over $H^{\prime}$ will be performed along the contour which goes from $-\rho$ to $\rho$ on the real axis, avoiding the poles of the exponential function, $H^{\prime}=$ $-b$ and $H^{\prime}=0$. The contour is closed by considering a large semicircle in the half part of the complex plane with negative imaginary part. This contour is represented in Fig. 2] It is equivalent to a small closed contour around the pole $H^{\prime}=H_{0}^{\prime}$ because of the Cauchy theorem. In addition, as $\rho \rightarrow \infty$, the integral along the large lower semicircle goes to zero and we are reduced to an integral from $-\infty$ to $+\infty$, as before. As announced above, this last argument justifies the fact that we have extracted the Green function from Eq. (55). Performing explicitly the integration, the Green function for topology change reads

$$
\begin{aligned}
G_{i}= & \frac{3 m y^{\prime} x^{5 / 2}}{4 \pi \hbar \kappa|v|\left(\chi-\chi^{\prime}\right)} \exp \left\{\frac{i}{\hbar \kappa}\left[-\frac{4 m\left(y-y^{\prime}\right)}{3\left(\chi-\chi^{\prime}\right)}+\frac{3 \mathcal{K} y^{1 / 3}\left(x^{4}-1\right)\left(\chi-\chi^{\prime}\right)}{10\left(x^{3}+1\right) x}\right]\right\} \exp \left\{\frac { 1 } { \kappa } \left[-\frac{8 m\left(y y^{\prime}\right)^{1 / 2}}{3\left(\chi-\chi^{\prime}\right)}\right.\right. \\
& \left.\left.-\frac{3 \mathcal{K} y^{1 / 3} x^{1 / 2}(x+1)\left(\chi-\chi^{\prime}\right)}{10\left(x^{3}+1\right)}\right]\right\} \Theta\left(-\frac{\mathcal{K}}{\mathcal{K}^{\prime}}\right),
\end{aligned}
$$

where the quantity $x$ has been introduced before. This is the main result of this section.

Let us now check that the above expression possesses the correct properties of a Green function. The WheelerDe Witt equation for the homogeneous background is given by

$$
-i m \hbar \frac{\partial \Psi}{\partial \chi}=-\frac{3 \kappa}{4} \hbar^{2} y \frac{\partial^{2} \Psi}{\partial y^{2}}-\frac{\mathcal{K} y^{1 / 3}}{2 \kappa} \Psi .
$$

The Green function $G_{i}$ must satisfy the semi-classical version of Eq. (57). Recovering the units, $G_{i}$ has the following form: $G_{i}=C \exp (i S / \hbar) \exp (A / \hbar)$. After inserting this expression in Eq. (57), one obtains an equation whose real and imaginary parts, in the limit $\hbar \rightarrow 0$, read

$$
m \frac{\partial S}{\partial \chi}-\frac{3 \kappa}{4} y\left[\left(\frac{\partial S}{\partial y}\right)^{2}-\left(\frac{\partial A}{\partial y}\right)^{2}\right]-\frac{\mathcal{K} y^{1 / 3}}{2 \kappa}=0
$$

$$
-m \frac{\partial A}{\partial \chi}+\frac{3 \kappa}{2} y \frac{\partial S}{\partial y} \frac{\partial A}{\partial y}=0
$$

Using the expression of $G_{i}$ derived above, see Eq. (56), one can easily check that Eqs. (58) and (59) are indeed satisfied in the long-wavelength approximation. Let us notice that terms involving $R^{2}=\left(6 \mathcal{K} / y^{2 / 3}\right)^{2}$ have been neglected. Hence, $G_{i}$ possesses all the good properties of a Green function signaling a topology change: it describes the transition from a wave function describing an homogeneous spatial geometry with positive (negative) curvature to another wave function describing another homogeneous spatial geometry with negative (positive) curvature [see Eq. [23)], and it satisfies the semi-classical Wheeler-De Witt equation in the long-wavelength approximation. 


\section{Discussion}

In this section, we briefly discuss the main properties of the Green function of Eq. (56).

First of all, it is interesting to notice that, without the change of sign obtained in the first term of the exponential, see Eq. (53), the Green function obtained through Eq. (23) would not satisfy the Wheeler-De Witt equation (57). This change of sign is a direct consequence of describing the change of topology by means of complexification of coordinates, which in turn implies an overall change of sign in the four-metric while the transition is taking place. This confirms the ideas of Ref. [13] stating that, in a topology transition, one should pass through degenerate metrics. We notice in passing that Eq. (58) is not exactly the classical Hamilton-Jacobi equation for a minisuperspace corresponding to the homogeneous metrics considered here. There is an extra term which induces the quantum change of topology. This is the quantum potential in the Bohm-de Broglie interpretation language. This extra term comes from the imaginary part $A$ of the action and indicates that such a change of topology is in fact a quantum tunneling effect.

Secondly, examining the Green function (56), one can notice that a topology change is very improbable if $\Delta \chi \equiv \chi-\chi^{\prime}$ is very short. As $\Delta \chi$ increases, quantum changes of topology become possible in both directions (from positive to negative curvature and vice-versa), but when $\Delta \chi$ becomes very large, negative to positive curvature transitions are suppressed while positive to negative curvature transitions are enhanced. This suggests that negative curvature topologies are preferred. Finally, for finite $\Delta \chi$, topology transitions between large volume spacelike hypersurfaces are very improbable, as expected.

\section{CONCLUSIONS}

We now briefly summarize the main results reached in this article.
We have obtained a Green function describing a topology change by using a double approximation: the semiclassical canonical quantum gravity and the long wavelength approximation. Our considerations have been restricted to transitions between homogeneous spacelike hypersurfaces with different intrinsic curvatures. In this framework, we have been able to demonstrate that transitions involving flat hypersurfaces are forbidden. However, we have also shown that this is no longer the case if the hypersurfaces are curved. In order to obtain the corresponding Green functions describing transitions between curved hypersurfaces, we have used imaginary coordinates (not only timelike coordinate but also one spacelike coordinate) in order to circumvent the midisuperspace problem raised in Ref. 4] (i.e. the components of the four-dimensional curvature tensor are only functions of time). The explicit expression of the Green function has been obtained by performing an integration in the complex plane. The resulting Green function shows that topology changes in the direction of negatively curved hypersurfaces are strongly enhanced as time goes on while transitions in the opposite direction are suppressed. In a finite amount of time, transitions between large spacelike hypersurfaces are improbable.

The formalism developed in this paper can also be applied to more complicated models. This will be the subject of our future investigations.

\section{ACKNOWLEDGMENTS}

Two of us (NPN and IDS) would like to thank the Cosmology Group of CBPF for useful discussions, and CNPq of Brazil for financial support.
[1] R. Geroch, J. Math. Phys. 8, 782 (1967).

[2] G. T. Horowitz, Class. Quantum Grav. 8, 587 (1991).

[3] D. H. Coule and J. Martin, Phys. Rev. D 61, 063501 (2000).

[4] V. A. De Lorenci, J. Martin, N. Pinto-Neto and I. Damião Soares Phys. Rev. D 56, 3329 (1997).

[5] M. Lachièze-Rey and J. P. Luminet, Phys. Report 254, 135 (1995).

[6] K. V. Kuchar and C. G. Torre, Phys. Rev. D 43, 419 (1991).

[7] J. B. Hartle and S. W. Hawking, Phys. Rev. D 28, 2960 (1983).

[8] D. S. Salopek and J. M. Stewart, Class. Quantum Grav. 9, 1943 (1992).
[9] D. S. Salopek and J. M. Stewart, Phys. Rev. D 47, 3235 (1993).

[10] J. Parry, D. S. Salopek and J. M. Stewart, Phys. Rev. D 49, 2872 (1994).

[11] D. S. Salopek, Phys. Rev. D 46, 4373 (1992).

[12] P. A. M. Dirac, Lectures on Quantum Mechanics (Yeshiva University, New York, 1964).

[13] S. W. Hawking, Euclidean Quantum Gravity (World Scientific, Singapore, 1993).

[14] J. M. Robbins, S. C. Creagh and R. G. Littlejohn, Phys. Rev. A 39, 2838 (1989); S. C. Creagh and N. D. Whelan, Phys. Rev. Lett. 77, 4975 (1996). 